\title{
Conocimientos en salud bucal de los cuidadores del Programa Buen Comienzo*
}

\section{Oral Health Knowledge of the Caregivers of the 'Buen Comienzo' Program}

\section{Conhecimentos em saúde bucal dos cuidadores do Programa Bom-Começo}

Fecha de recepción: 10-05-16 Fecha de aceptación: 28-07-16 Disponible en línea: 30-11-16 doi:10.11144/Javeriana.rgyps15-31.csbc

Cómo citar este artículo:

González-Penagos C, Cano-Gómez M, Meneses-Gómez EJ, Saldarriaga-Bolívar V. Conocimientos en salud bucal de los cuidadores del programa Buen Comienzo. Rev. Gerenc. Polít. Salud. 2016; 15(31):

130-144. http://dx.doi.org/10.11144/Javeriana.rgyps15-31.csbc

\author{
Catalina González-Penagos** \\ Melissa Cano-Gómez*** \\ Edwin J. Meneses-Gómez $* * * *$ \\ Verónica Saldarriaga-Bolívar*****
}

\footnotetext{
Artículo de investigación, producto del proyecto de investigación Caracterización de las comunidades y análisis de las representaciones de necesidades en salud bucal de colectivos Buen Comienzo de la ciudad de Medellín, fase II 2015, código: INV- P19. Ejecutado en el periodo de febrero del 2015 a marzo del 2016 por el grupo de investigación GISCo (Grupo de Investigación en Salud y Comunidad) y financiado por la Fundación Universitaria Autónoma de las Américas, Medellín, Colombia.

** Autor principal y de correspondencia. Profesional en Desarrollo Familiar, especialista en Docencia Investigativa Universitaria, magíster en Salud Pública, profesora investigadora de la Facultad de Odontología de la Fundación Universitaria Autónoma de las Américas y del Grupo de Investigación en Salud y Comunidad. Dirección: Calle 34A No.76-35, Medellín, Colombia. Correo electrónico: catalina.gonzalez@uam.edu.co

*** Auxiliar de investigación. Estudiante de décimo semestre de la Facultad de Odontología de la Fundación Universitaria Autónoma de las Américas e integrante del Grupo de Investigación en Salud y Comunidad. Correo electrónico: melissacanogomez@gmail.com

***** Odontólogo, especialista en Odontología Preventiva y Comunitaria, Ph. D. en Ciencias Odontológicas, profesor investigador de la Facultad de Odontología de la Fundación Universitaria Autónoma de las Américas y del Grupo de Investigación en Salud y Comunidad. Correo electrónico: edwin.meneses@uam.edu.co

***** Odontóloga, especialista en Docencia Investigativa Universitaria, profesora investigadora de la Facultad de Odontología de la Fundación Universitaria Autónoma de las Américas y del Grupo de Investigación en Salud y Comunidad. Correo electrónico: verónica.saldarriaga@uam.edu.co
} 


\section{Resumen}

Objetivo: analizar los conocimientos en salud bucal de los cuidadores del Programa Buen Comienzo, Fantasías de las Américas, 2015. Materiales y métodos: estudio cualitativo históricohermenéutico, la información se obtuvo a partir de la técnica interactiva descriptiva del mural de situaciones. Población conformada por 183 padres, madres y cuidadores de los Centros Infantiles Fantasías de las Américas. Se identificaron unidades de análisis y se crearon categorías y subcategorías, con el fin de reducir los datos y realizar la contrastación y la triangulación de la información. Resultados: se evidenció una pluralidad en las necesidades expresadas por los cuidadores en relación con la salud bucal y aspectos relacionados con su prevención y tratamiento. Se identificaron necesidades específicas relacionadas con patologías y condiciones asociadas a los hábitos, así como las alteraciones en la variabilidad de la salud bucal. Conclusiones: la identificación de las necesidades expresadas y sentidas por los cuidadores exige que se tengan en cuenta nuevas temáticas para enriquecer los saberes desde las actividades de educación y promoción de la salud. Se abre una mirada pluralista en la que todos participan, conocen, discuten y exigen calidad.

Palabras clave: infante; salud bucal; cuidadores; educación; conocimiento; evaluación de necesidades

\section{Abstract}

Objective: analyzing the knowledge on oral health of the caregivers of the 'Buen Comienzo' program, 'Fantasias de las Americas', 2015. Materials and Methods: historical-hermeneutical qualitative study. The information was obtained with the descriptive interactive technique of situation mural. The population was comprised of 183 parents, mothers, and caregivers of the children's center 'Fantasias de las Americas'. We identified analysis units and created categories and subcategories with the purpose of reducing data, contrasting and triangulating the information. Results: we evidenced plurality in the needs expressed by the caregivers regarding oral health and the aspects related to prevention and treatments. We identified specific needs related to pathologies and conditions associated to habits, as well as to the alterations in the variability of oral health. Conclusions: the identification of the needs expressed and felt by caregivers requires new topics to be taken into account as to enrich the knowledge from education and health promotion activities. We give way to a pluralist outlook where everyone participates, learns, discusses, and demands quality.

Keywords: children; oral health; caregivers; education; knowledge; needs assessment

\section{Resumo}

Objetivo: analisar os conhecimentos em saúde bucal dos cuidadores do Programa Buen Comienzo, Fantasías de las Américas (Bom- Começo, Quimeras das Américas), 2015. Materiais e métodos: estudo qualitativo histórico-hermenêutico. A informação foi obtida a partir da técnica interativa descritiva de mural de situações. População conformada por 183 pais, mães e cuidadores dos Centros Infantis Quimeras das Américas. Identificaram-se unidades de análise e criaram-se categorias e subcategorias a fim de reduzir os dados e realizar o contraste e triangulação das informações. Resultados: evidenciou-se uma pluralidade nas necessidades expressas pelos cuidadores em relação com saúde bucal e aspectos relacionados com prevenção e tratamento. Foram identificadas necessidades específicas relacionadas com patologias e condições associadas a hábitos, bem como as alterações na variabilidade da saúde bucal. Conclusões: a identificação de necessidades expressas e sentidas pelos cuidadores exige levarem em conta novas temáticas para enriquecer os saberes desde as atividades de ensino e promoção da saúde. Abre-se um olhar pluralista em que todos participam, conhecem, discutem e exigem qualidade.

Palavras-chave: criança; saúde bucal; cuidadores; educação; conhecimento; avaliação de necessidades 


\section{Introducción}

En la actualidad, el trabajo por la primera infancia es un tema de gran importancia en la agenda política, económica y social, especialmente en países que se encuentran en vía de desarrollo, como Colombia. En respuesta a objetivos de orden mundial, se crean programas dirigidos específicamente a los niños y las niñas, quienes conforman una de las poblaciones más vulnerables del país, y se reconoce la importancia que tiene intervenir en los menores durante los primeros años de vida, para lograr un adecuado desarrollo cognitivo y emocional que les permita, en esta etapa del ciclo vital, desenvolverse adecuadamente y lograr ser ciudadanos de bien en el futuro. Para alcanzar esta meta, se implementan diferentes tipos de intervenciones en la primera infancia, las cuales se enfocan en brindar apoyo a los niños y las niñas en las instituciones educativas y en los centros infantiles, así como hacer acompañamiento a las personas que son parte de su círculo social, como lo son los padres, los cuidadores y los agentes educativos, con el fin de mejorar la calidad y el proceso de crecimiento y desarrollo de este colectivo $(1,2)$.

Todos los esfuerzos dirigidos a la atención integral a la primera infancia se orientan hacia la calidad de su cuidado en los diferentes contextos sociales y sus diversas representaciones, con el fin de proporcionar un comienzo solido en la vida, gracias a la atención y la educación por parte de los cuidadores, pues los desafíos económicos y sociales de la globalización así lo exigen (3). Por esa razón, se implementan estrategias de Atención Integrada a las Enfermedades Prevalentes de la Infancia (AIEPI), para poder cumplir con los diferentes objetivos nacionales e internacionales (4). lud de las personas en las diferentes regiones de un país, antes de realizar cualquier tipo de intervención. Inicialmente, es necesario orientarse con base en los niveles de conocimiento que se tienen sobre la etiología y la prevención de estas alteraciones, así como las prácticas diarias de higiene y la actitud al respecto. (5) Además, al indagar en niños y niñas menores de cinco años, cualquier solicitud debe ir dirigida a las personas encargadas de su cuidado, tal como lo recomiendan las Normas Técnicas de Atención Preventiva en Salud Bucal del Sistema General de Seguridad Social en Salud (SGSS) (6).

Para dar cumplimiento a la política pública nacional Colombia para la Primera Infancia, del año 2007, así como a programas estatales como la estrategia De Cero a Siempre, de la Consejería Presidencial para la Primera Infancia, que busca de manera intersectorial asegurar el acceso de los menores a la educación, la salud, la nutrición y la recreación, se crea el programa regional Buen Comienzo, de la Secretaría de Educación de Medellín. En este se incluye el entorno familiar, que es el primer ente educador de los menores, y el entorno institucional y comunitario, donde participan los agentes educativos durante los primeros cinco años de vida de los niños y las niñas, para lograr su desarrollo integral, diverso, incluyente y autónomo (7).

Una de las metas del programa es lograr condiciones óptimas de salud en los niños y las niñas. Para alcanzarla, resulta evidente la importancia de la dimensión de la salud en el componente bucal, como se manifiesta en los lineamientos y estándares para la prestación del servicio de atención integral a la primera infancia del Programa Buen Comienzo $(8,9)$, donde se enfatiza que la salud bucal cumple un papel significativo en el desarrollo cráneofacial y es indispensable para una adecuada fonación y nutrición de los menores. 
Para llevar a cabo este lineamiento, la Facultad de Odontología establece una alianza de trabajo con el operador del Programa Buen Comienzo Fantasías de las Américas de la Fundación Universitaria Autónoma de las Américas, y se implementan estrategias de promoción y prevención de la salud bucal mediante la educación continua y la recreación que incluyen reuniones grupales dirigidas a los niños, las niñas y los adultos significativos. Estos espacios permiten identificar problemáticas y necesidades en salud bucal desde las percepciones que los mencionados grupos tienen sobre su realidad.

Las percepciones, obtenidas mediante técnicas interactivas, arrojan resultados que varían a través del tiempo, pero son susceptibles a intervenciones que pueden lograr cambios significativos en las condiciones de inequidad y desigualdad en las que se encuentra esta población, lo cual permite que los niños y las niñas desempeñen un papel incluyente en su núcleo familiar actuando como vigías de salud bucal. La evidencia científica muestra que la salud bucal de los niños se encuentra muy relacionada con el periodo gestacional de la madre, ya que las alteraciones que se presentan durante este periodo pueden afectar la salud bucodental del hijo. Asimismo, se considera que algunos factores de riesgo se encuentran asociados a esta problemática, entre ellos condiciones sociales como el nivel socioeconómico y el nivel educativo y cultural de la familia (10).

Debido a dificultades similares a las mencionadas, los gobiernos y diferentes organizaciones trabajan para disminuir la morbilidad y la alta prevalencia de la caries dental, para lo cual se implementan estrategias y políticas públicas que permitan hacer un abordaje integral y total de la población en riesgo. En diciembre del 2015, la ciudad de Medellín aprobó la Política Pública de Salud Bucal
2013-2022, establecida entonces como meta en el marco del Plan Territorial de Salud 2012-2015 (11).

La política en mención define algunas estrategias importantes, a través de las cuales se motiva a una participación social y un empoderamiento comunitario que logre eliminar barreras y condiciones de inequidad que afectan la salud comunitaria. Esta política pública es incluyente y considera la participación de todos los involucrados en temas relacionados con el componente bucal de la salud. Igualmente, genera un gran impacto, ya que se tiene en cuenta a todos los adultos significativos que actúan, como pilares al momento de implementar hábitos y prácticas saludables en la cotidianidad de los menores.

Las actividades realizadas con la población de los Centros Infantiles Fantasías de las Américas del Programa Buen Comienzo cumplen con uno de los objetivos propuestos en la política pública en salud bucal de Medellín, esto es, la integración de los profesionales y los odontólogos en proceso de formación, de manera activa y participativa, en todos los temas relacionados con salud pública en la ciudad. Esta participación permite modificar y mejorar percepciones y conocimientos acerca de la salud bucal que existen en el imaginario de las poblaciones vulnerables, lo cual permite lograr mejoras en la calidad de vida de las personas. Al realizar cambios positivos en ese imaginario, las comunidades se empoderan de sus derechos fundamentales y se cambia la percepción en los colectivos acerca de que la salud bucodental está aislada de la salud general.

De acuerdo a lo anterior, se propone desarrollar esta investigación con el objetivo de analizar los conocimientos en salud bucal de los cuidadores del Programa Buen Comienzo, Fantasías de las Américas, 2015. A partir de estos resultados se puede promover 
la realización de nuevas investigaciones en relación con el tema de la salud bucal que aporten a la disminución de los factores de riesgo de esta en la primera infancia.

\section{Materiales y métodos}

Se llevó a cabo un estudio cualitativo, con enfoque histórico-hermenéutico, el cual permitió comprender e interpretar la realidad y el sentido que le dan los cuidadores de los niños y las niñas a los conocimientos en salud bucal en un contexto concreto. La investigación cualitativa posibilita la utilización de diversas estrategias metodológicas y fuentes, lo cual potencia la complementariedad de los métodos. En esta investigación, a diferencia de las técnicas que se usan tradicionalmente, se privilegian las técnicas interactivas para la investigación social cualitativa. De esta manera, se obtuvo y se analizó la información a partir de la utilización de la técnica interactiva descriptiva del mural de situaciones, la cual permite la activación y la expresión de las personas, generando procesos interactivos que promueven el reconocimiento y el encuentro entre los sujetos; esto, además, propicia la construcción colectiva del conocimiento (12). También se utilizó la observación como técnica que trasciende la simple descripción de la situación y permite la identificación del sentido, la orientación y la dinámica de cada instante (13).

La Fundación Universitaria Autónoma de las Américas, con la Facultad de Odontología y en alianza con el Centro de Desarrollo Social, dependencia que materializa la extensión y la proyección social de la Universidad, y como oferente de 8 Centros Infantiles Fantasías de las Américas Institucional 8 horas, ubicados en diferentes comunas de 134 Medellín, y que son parte del Programa Buen Comienzo, se propone fortalecer de manera prioritaria el componente bucal con el trabajo en las comunidades del Programa.

De acuerdo a lo anterior, la población estuvo conformada por 183 padres, madres o cuidadores de tres de los centros infantiles Institucional 8 horas Fantasías de las Américas del Programa Buen Comienzo en el año 2015, programa de la Alcaldía de Medellín que promueve el desarrollo integral, diverso e incluyente de niños y niñas. Los participantes fueron informados previamente de los objetivos de la investigación, de sus derechos, así como también del procedimiento y el tiempo en que se llevaría a cabo. Por medio de un consentimiento informado, diligenciado por los padres, las madres y los cuidadores de los niños y las niñas, se hicieron las aclaraciones del proceso realizado, con el fin de que tuvieran una comprensión sobre su participación voluntaria en la investigación.

Para el estudio y la interpretación de los datos se contempló la descripción de las características sociodemográficas, institucionales y del entorno en el cual se encuentran las comunidades del programa Buen Comienzo (Institucional 8 Horas), además del análisis de los conocimientos en salud bucal de los cuidadores del programa. Con base en ello, la investigación se desarrolló a través de varias fases.

En un primer momento, se llevó a cabo la fase de exploración y consenso y se realizó la recopilación de la información para la preparación de la investigación, en donde se sistematizaron los conocimientos obtenidos durante el acercamiento al tema, incluyendo el planteamiento del problema, la justificación, los objetivos, la memoria metodológica y el cronograma de trabajo. Asimismo, se establecieron las técnicas de recolección de información y se delimitaron los componentes de las técnicas y los criterios de selección para cada una de ellas. Finalmente, se hizo 
la planeación de las actividades que se ejecutaron en las fases posteriores.

Un segundo momento fue el de la contextualización. En este, en primer lugar, se hizo un reconocimiento del campo en el cual se iba a recoger la información, lo cual permitió asegurar el éxito de la investigación, además del reconocimiento de las comunidades del Programa Buen Comienzo. Además, se utilizó la observación como un proceso transversal en la investigación, se hizo la caracterización de la población, la caracterización institucional y la caracterización del entorno de las comunidades de Buen Comienzo.

En un tercer momento, se pasó a la fase de profundización, la cual tuvo como propósito organizar y aplicar las técnicas con la población seleccionada. El equipo de trabajo organizó el diseño de las técnicas y las puso a prueba, para que finalmente, luego de ser ajustadas, se pudieran aplicar mediante el contacto directo con la población.

Para el estudio y la interpretación de los datos se identificó la disgregación de las unidades de análisis, específicamente en líneas de textos individuales y grupales, lo que permitió la ordenación conceptual y la utilización de códigos específicos que facilitaron la agrupación de la información en categorías. Además, se crearon subcategorías para realizar una interpretación detallada (ver tabla 1). Este proceso se estableció para reducir los datos y realizar la contrastación y triangulación de la información, con el fin de profundizar y consolidar la información obtenida.

Esta investigación cumplió con los requisitos éticos para investigación en salud contemplados en la Resolución 8430 de 1993 del Ministerio de la Salud y Protección Social de Colombia y se clasifica como una investigación sin riesgo (14). Fue avalada por el Centro de Desarrollo Social de la Fundación Universita- ria Autónoma de las Américas, como oferente del Programa Buen Comienzo de la ciudad de Medellín y contó con la aprobación del Comité de Ética en Investigación (CEI) de la Universidad Autónoma de las Américas.

\section{Resultados}

Al analizar la información recolectada se evidenció una pluralidad en las necesidades expresadas por padres, madres y cuidadores en relación con la salud bucal y los aspectos relacionados con su prevención y tratamiento. Las personas identificaron necesidades específicas relacionadas con patologías y condiciones asociadas a los hábitos, así como las alteraciones en la variabilidad de la salud bucal. El análisis de los resultados permitió identificar dieciocho categorías (tabla 1), las cuales se exponen a continuación, y al final de cada una de ellas se cita en líneas individuales lo expresado por algunos de los cuidadores.

\section{Categorías}

Hábitos de higiene. Las personas enfatizaron en tener claridad para realizar el control de hábitos como el consumo de la crema dental o el cepillado excesivo y se preocuparon por conocer las técnicas correctas de cepillado y la limpieza de la lengua. Mencionaron que, en ocasiones, los niños y las niñas presentan halitosis, asociada particularmente al descuido en la higiene de la lengua. De la misma forma, se observó inquietud por la importancia del correcto lavado de las manos como parte integral de un proceso de higiene:

- Cepillarse todos los días mañana y noche cuando uno ya se va a acostar, hasta el otro día. Gracias a Dios coge el cepillo se lava la boca y todo. 
TABla 1. Descripción de Códigos, Categorías y SubCATEgorías

\begin{tabular}{|c|c|c|c|}
\hline $\mathrm{N}^{\circ}$ & Código & Categoría & Subcategoría \\
\hline 1 & $\mathrm{HH}$ & Hábitos de higiene & $\begin{array}{l}\text { Consumo de crema dental, cepillado excesivo, } \\
\text { lavado de la lengua, lavado de manos, técnicas } \\
\text { correctas de cepillado. }\end{array}$ \\
\hline 2 & $\mathrm{IH}$ & Implementos de higiene & $\begin{array}{l}\text { Enjuague bucal, consumo dentríficos, lugar de } \\
\text { ubicación de los implementos, dentríficos con } \\
\text { flúor. }\end{array}$ \\
\hline 3 & SSB & Servicios de salud bucal & $\begin{array}{l}\text { Jornadas de salud bucal, odontopediatras en el } \\
\text { Plan Obligatorio de Salud (POS). }\end{array}$ \\
\hline 4 & $\mathrm{HO}$ & Hábitos orales & Besos y transmisión de infecciones. \\
\hline 5 & $\mathrm{CD}$ & Caries dental & Cómo prevenir la caries y su tiempo de aparición. \\
\hline 6 & $\mathrm{EP}$ & $\begin{array}{l}\text { Enfermedades del } \\
\text { periodonto y tejidos } \\
\text { blandos }\end{array}$ & $\begin{array}{l}\text { Cálculo dental, heridas y úlceras. Tratamiento al } \\
\text { mal aliento. }\end{array}$ \\
\hline 7 & AP & $\begin{array}{l}\text { Acompañamiento de los } \\
\text { padres }\end{array}$ & Jugar al odontólogo. \\
\hline 8 & $\mathrm{AOA}$ & $\begin{array}{l}\text { Alteraciones oclusales } \\
\text { autopercibas }\end{array}$ & Mordida abierta. \\
\hline 9 & ASB & $\begin{array}{l}\text { Alimentación y salud } \\
\text { bucodental }\end{array}$ & $\begin{array}{l}\text { Efectos del sulfato ferroso, consumo de calcio, } \\
\text { pan como cariogénico. }\end{array}$ \\
\hline 10 & OA & $\begin{array}{l}\text { Otras alteraciones } \\
* \text { No incluye caries }\end{array}$ & $\begin{array}{l}\text { Fracturas, pérdida temprana de la dentición por } \\
\text { trauma, restos radiculares y dolor, sensibilidad, } \\
\text { causas. }\end{array}$ \\
\hline 11 & NT & $\begin{array}{l}\text { Necesidades de } \\
\text { tratamiento }\end{array}$ & Aplicación de flúor y sellantes. \\
\hline 12 & $\mathrm{PC}$ & Procedimientos clínicos & $\begin{array}{l}\text { Aplicación de anestesia, limpiezas, ortodoncia y } \\
\text { cambios de color, operatoria. }\end{array}$ \\
\hline 13 & OS & $\begin{array}{l}\text { Odontología y salud } \\
\text { general }\end{array}$ & $\begin{array}{l}\text { Amigdalitis y salud bucal, boca y cáncer, embarazo } \\
\text { y salud bucal, dificultades en la fonación, emesis, } \\
\text { erupción dental y síntomas asociados. }\end{array}$ \\
\hline 14 & MS & $\begin{array}{l}\text { Medicamentos y salud } \\
\text { bucal }\end{array}$ & Efectos de los medicamentos. \\
\hline 15 & B & Bruxismo & $\begin{array}{l}\text { Edad de aparición, identificación de la } \\
\text { parafunción. }\end{array}$ \\
\hline 16 & $\mathrm{AD}$ & $\begin{array}{l}\text { Alteraciones del } \\
\text { desarrollo }\end{array}$ & $\begin{array}{l}\text { Dientes natales, erupción retardada, apiñamiento } \\
\text { y herencia, anodoncias. }\end{array}$ \\
\hline 17 & $\mathrm{RD}$ & Recambio dental & Edades de recambio, limitación en adultos. \\
\hline 18 & ATM & $\begin{array}{l}\text { Articulación temporo } \\
\text { mandibular }\end{array}$ & Limitación en apertura, ruidos. \\
\hline
\end{tabular}


- ¿ ¿Cuál es la mejor forma de lavar la lengua de los niños sin que cause mucha molestia?

- ¿Es cierto que la lengua se puede cepillar?

- ¿Por qué es necesario lavar bien la lengua?

- ¿El aseo de las manos cómo influye?

- ¿Porque el niño tiene mal olor a pesar de cepillarlo?

- ¿Cómo contrarrestar el mal aliento?

- ¿El mal aliento es falta de buen cepillado?

- (Preguntas textuales hechas por los padres y cuidadores durante la técnica interactiva Mural de Situaciones)

Implementos de higiene. En relación con el uso de los elementos de apoyo a la higiene bucal, se menciona al enjuague como uno que genera confusión acerca de si es pertinente o no utilizarlo a tan temprana edad. Adicionalmente, se manifestó inquietud por el lugar de la casa o del baño donde se deben ubicar los implementos de higiene utilizados:

- ¿Un niño de cinco años puede usar enjuague y crema dental normal?

- iEs bueno el enjuague bucal para el menor?

- iEs conveniente tener los implementos de higiene bucal en el baño?

- ¿Qué tan cierto es que no es conveniente tener los implementos de salud bucal en el baño?

(Preguntas textuales hechas por los padres y cuidadores durante la técnica interactiva Mural de Situaciones)

Hábitos orales, caries dental y enfermedades del periodonto y tejidos blandos. En la información recogida se expresan dudas en relación con los besos entre padres e hijos, la comprensión de si hay o no posibilidad de transmitir infecciones. Los adultos con quienes se realizó el trabajo reconocen la caries como una enfermedad propia de los tejidos dentales, pero se inquietaron por entender cómo es posible prevenirla, pues la consideran una condición común, y también enfatizaron en reconocer su tiempo de aparición. Otras de las patologías que identificaron son las lesiones de los tejidos blandos, heridas y úlceras, con respecto a las cuales necesitan algunas medidas para tratarlas, pues mencionan que son de común aparición, tanto en los niños como en los adultos:

- ¿Es malo dar besos a los niños en la boca?

- ¿En los besos que le damos a los niños se transmiten bacterias?

- ¿Es verdad que hay medicamentos que ayudan a dar caries?

- ¿Cómo puede uno evitar las enfermedades de la salud bucal o la caries a los niños?

- ¿Por qué les da caries a tan temprana edad?

- La niña mía no se deja cepillar de mí, quiere ella solita, pero no se limpia bien y le dieron caries.

- ¿ ¿Las caries en qué tiempo aparecen en los niños?

- ¿Cómo puedo prevenir la aparición de las caries?

- ¿Por qué a un niño no se le pican tan fácil las muelitas? ¿De qué otra manera se puede prevenir las caries a parte del cepillado?

- ¿Por qué a veces salen llagas en la boca?

- ¿Qué produce las llagas?

- ¿Quisiera saber por qué dan llagas y cuando esto pasa cómo debemos cepillar para no lastimar?

(Preguntas textuales hechas por los padres y cuidadores durante la técnica interactiva Mural de Situaciones)

Acompañamiento de los padres. Algunas madres priorizaron la importancia del acercamiento de los niños a la salud bucal por medio del juego, en aquellos casos que tienen 
dificultades para que los menores acepten la primera consulta o el tratamiento odontológico. En este sentido, explicaron que ellas mismas simulan la atención en casa, jugar al odontólogo y reconocerlo.

- Le juego al odontólogo. La salud bucal es importante, tener acceso a ella ya que se previenen enfermedades en la boca y mal olor. Es importante cuidar los dientes ya que de ahí depende la presentación de cada persona.

(Preguntas textuales hechas por los padres y cuidadores durante la técnica interactiva Mural de Situaciones)

Otras alteraciones. Cuando se presentan inquietudes relacionadas con esta categoría, algunos padres indagan sobre el manejo que se debe dar a los restos radiculares, pues son motivo frecuente de dolor. A su vez, se evidencia que la presencia de sensibilidad es una queja común, y los padres quieren conocer la causa que genera esta condición. Mencionan los frecuentes traumatismos en los niños y niñas, con la consecuente pérdida temprana de la dentición:

- ¿Cuándo uno tiene raíces de las muelas por qué duelen tanto y eso genera mal aliento?

- ¿Por qué se vuelven sensibles los dientes al comer cosas heladas y duras?

- ¿Por qué a uno le da tanta sensibilidad en los dientes?

- Mi nieto cuando gateaba se golpeó un dientecito ahora se puso gris. iEstá flojo!

- ¿Qué se le hace cuando a un niño se tumba un diente de leche antes de tiempo?

(Preguntas textuales hechas por los padres y cuidadores durante la técnica interactiva Mural de Situaciones)

Necesidades de tratamiento. Los padres tienen un particular acercamiento y dominio de conceptos relacionados con los tratamientos. Reco- nocen la existencia de la aplicación del flúor y los sellantes, se interrogan sobre su importancia, cuál es la frecuencia con que se debe realizar y qué condiciones la indican o la recomiendan.

- A mi hija le descubrieron que tenía una caries en la mitad de los dos dientecitos, pedí la cita la llevé donde el odontopediatra, le limaron los dientecitos y hurgaron en ellos por un rato, luego le colocaron una pequeña pasta blanca y ya estaba lista. A los días esta pequeña pasta se le cayó y la niña quedó con un hueco en la mitad de los dientes volví a pedir cita le hicieron el mismo procedimiento, el odontólogo dijo que era una bobada.

- ¿Desde qué edad se recomienda el uso de sellantes?

- ¿Qué tan recomendable es el uso de la crema dental en los niños? (Colgate) así sea sin flúor?

- Tengo una niña de cuatro años de edad y fue demasiado propensa a las caries y sobre todo le empezó porque las fisuritas de los molares eran muy profundas. Ella se cepillaba normalmente pero ahí siempre quedaba algo más que todo cuando comía dulces, galletas, etc. y mi recomendación es estar muy pendientes de los sellantes.

- ¿Cada cuánto se debe aplicar el flúor en los dientes de los niños?

- ¿Hasta qué edad se debe utilizar la crema dental sin flúor en los niños?

- ¿Desde qué edad se recomienda el uso de sellantes?

(Preguntas textuales hechas por los padres y cuidadores durante la técnica interactiva Mural de Situaciones)

Procedimientos clínicos. Se cuestiona la aplicación de la anestesia y su efectividad en los menores:

- ¿A un niño se le puede poner anestesia para sacarle un diente? 
(Preguntas textuales hechas por los padres y cuidadores durante la técnica interactiva Mural de Situaciones)

Alteraciones oclusales autopercibidas y bruxismo. Algunas familias mencionan cambios evidentes en las alteraciones de tipo oclusal, como la mordida abierta y el apiñamiento, y hacen preguntas sobre el tratamiento de ortodoncia temprano. Se preguntan en particular por la relación que existe entre la herencia y estas condiciones. También se refieren a la aparición del bruxismo, sus características y su prevención:

- ¿ ¿Qué se puede hacer para arreglar una mordida abierta, porque hay niños con mordida abierta?

- Mi hijo tiene mordida cruzada ¿Este problema se resuelve a medida que él va creciendo? ¿O tendré que hacerle tratamiento cuando tenga los dientes definitivos?

- ¿Por qué los niños no se le pueden poner braquets?

- ¿Es normal el bruxismo en un niño menor de cinco años?

- Algunos no saben qué es el bruxismo.

- $\quad$ Siempre que esto se hace (el bruxismo) siempre es bruxismo o hay un punto que el bruxismo es por lombrices.

- Pero entonces sería bueno consultar cuando hay bruxismo.

- Una madre que estaba con su bebe al lado dice: "Esta bebé desde que le empezaron a crecer los dientes le traquean, pero no es todo el tiempo entonces es que le están creciendo los dientes o si no entonces porque, casi siempre cuando esta despierta".

- Una mama dice: "Algunas nos quedó inconcluso lo del bruxismo, se debe consultar cuando están muy desgatados y funcionan normal? o sea, los tienen planitos y también le da sensibilidad".
- ¿Es normal el crujir los dientes mientras se duerme?

- ¿Cuál es el motivo de chasquear los dientes en la noche tanto niño como adulto?

- ¿Es verdad que si el niño apretaba los dientes era por parásitos?

(Preguntas textuales hechas por los padres y cuidadores durante la técnica interactiva Mural de Situaciones)

Odontología, salud general, medicamentos y salud bucal, alteraciones del desarrollo. Algunas madres en periodo de gestación se inquietan por los cuidados que deben tener durante el embarazo para preservar la integridad de los dientes, así como por el efecto que tienen las emesis frecuentes:

- Cuando estaba en embarazo me dañaron las muelas y después en el otro embarazo volví a la misma doctora y me pregunto quién me había dañado las muelas y yo le dije que era ella y no lo reconoció.

- Después de vomitar qué tan obligatorio es el cepillo.

(Preguntas textuales hechas por los padres y cuidadores durante la técnica interactiva Mural de Situaciones)

Se evidencia un consenso y un interés particular por la relación entre la salud bucal y la salud general. Se observa que quieren ampliar su conocimiento en relación con la amigdalitis, el cáncer, las dificultades en la fonación y los efectos de los medicamentos sobre los dientes, particularmente en las alteraciones del desarrollo. Durante las sesiones de trabajo nombraron conceptos para profundizar en ellos, como los dientes natales, la erupción retardada, las dificultades en la fonación y las anodoncias:

- ¿Qué hacer cuando se presenta amigdalitis constantemente? 
- $\quad$ ¿La amigdalitis en un niño de cinco años es normal?

- ¿Qué se puede hacer cuando es muy constante la amigdalitis en un niño?

- ¿Es cierto que una infección en la boca puede causar la muerte o cáncer?

- ¿Es verdad que hay medicamentos que ayudan a dar caries o a dañar los dientes?, ¿cuáles son?

- ¿Por qué hay dientes que no nacen?

(Preguntas textuales hechas por los padres y cuidadores durante la técnica interactiva Mural de Situaciones)

\section{Recambio dental y articulación temporoman-} dibular. Dos categorías particularmente interesantes en este ejercicio con padres, madres y cuidadores. Los adultos indagan acerca de los motivos por los cuales a su edad actual no hay posibilidad de recambio dental. En su imaginario, desconocen la edad exacta en la que los niños tienen exfoliación de dientes deciduos y erupción de permanentes. Identifican la existencia y la función de la articulación temporomandibular, pero muestran interés en conocer los motivos por los cuales pueden tener limitación en la apertura y los ruidos que aparecen durante la función. Se observa inquietud por su oportuno tratamiento:

- Inquietud que a veces cuando abre o cierra le suena o le duele.

- Cuando se les caen los dientes.

- ¿A qué edad se caen por primera vez los dientes en niños menores de cinco años?

- En este momento tengo un niño de la edad de cinco años y en este momento le salió un diente y no sé si le tengo que arrancar el de leche o si lo tengo que llevar al odontólogo.

- ¿Cómo debo manejar la etapa en la que los niños se le caen los dientes para que los nuevos sean saludables y organizados?
(Preguntas textuales hechas por los padres y cuidadores durante la técnica interactiva Mural de Situaciones)

Servicios de salud bucal. Finalmente, durante el trabajo de campo realizado se hace evidente, por los diálogos sostenidos, que se requiere la presencia de odontopediatras en los servicios de salud de las instituciones prestadoras, debido a las múltiples y complejas situaciones bucodentales que presentan los menores. La comunidad o colectivo hace un llamado para que el Estado se apropie de esta situación y garantice mejores condiciones de acceso y calidad en los servicios de salud odontológicos y mejores profesionales para una atención de calidad:

- Uno lleva el niño y no lo revisan bien, no le hacen ni siquiera limpieza bucal solo lo miran y ya.

- Necesitamos que nos puedan atender cuando necesitamos y nos den buenas citas a tiempo para poder solucionar nuestros problemas bucales a tiempo y los de nuestros hijitos.

- La mala atención en el Sisben que nunca están los odontólogos.

- Teniendo en cuenta las situaciones económicas por que no hay odontopediatras en las EPS, ya que es más caro a veces y no se puede por la situación económica, sale más caro porque tiene que ser particular.

(Preguntas textuales hechas por los padres y cuidadores durante la técnica interactiva Mural de Situaciones)

\section{Discusión}

Los hallazgos hechos tras el trabajo de campo presentan una realidad que tiene unas particularidades sobre las cuales es necesario hacer algunas precisiones. De esta manera, va a ser posible establecer puntos 
de discusión que contribuyan a entender las percepciones de los padres de familia y de los cuidadores en general, como responsables de esta población de primera infancia, dependiente de los adultos.

En la literatura científica se encuentran algunos estudios relevantes que pueden aportar a la discusión, pero se evidencia que son más los estudios realizados con enfoques cuantitativos que los que aportan al conocimiento con metodologías similares a las del trabajo realizado. Dan cuenta de esto los trabajos del grupo de profesionales de la Universidad de Antioquia del 2009 y del 2010, basados en técnicas de teoría fundada, que si bien limitan su estudio a madres gestantes, destacan el papel de estas en el cuidado posterior de sus hijos (15). Un segundo trabajo publicado por la misma institución resalta la necesidad de trascender el imaginario del acto clínico como una relación objeto-objeto, reducida a la utilización de técnicas, y entender a los menores estableciendo actitudes de cuidado (16).

En los últimos años, es notorio el incremento de estudios orientados a profundizar la comprensión de las condiciones relacionadas con la primera infancia y sobre temas relativos al componente bucal de la salud. En el año 2007 se realizó en California un estudio con cuidadores de niños de uno a cinco años, para identificar creencias, prácticas y experiencias que puedan influir en el acceso preventivo a los servicios de salud bucal por parte de los menores. El estudio concluyó que hay variabilidad por grupos raciales, con algunas similitudes entre unos y otros como la falta de conocimiento y algunos mitos que crean barreras al acceso a la atención preventiva (17).

Otros estudios han revelado creencias y conocimientos sobre la etiología de la caries que evidencian gran confusión entre las madres sobre conceptos específicos, los cuales, igualmente, invitan a la creación de estrategias para una educación más amplia (18).

Para el año 2010, en Ámsterdam se habían realizado estudios relacionados con la necesidad de categorizar los conocimientos de los padres en relación con la salud bucal de sus hijos. Estos conocimientos tienen que ver con actitudes y opiniones que en su complejidad pueden permitir la interpretación de necesidades y la toma de decisiones para crear estrategias de prevención (19).

Estos hallazgos permiten una aproximación a las percepciones, los conocimientos, las prácticas y, sobre todo, el impacto de los programas a través de los cuales se busca desarrollar las políticas nacionales e internacionales para contribuir a mejorar las condiciones de salud de los menores y, especialmente, el papel de los cuidadores, e incluso del núcleo familiar completo, como responsables del proceso.

El reciente Estudio Nacional de Salud Bucal Ensab IV presenta resultados que deben ser tenidos en cuenta, y hacer un seguimiento y monitoreo permanente de colectivos vulnerables como la primera infancia, pues muestran un notable aumento de los indicadores relacionados con la salud bucal presentados en el reciente estudio (ENSAB IV), los cuales quizá siempre han sido objeto de atención, pero hoy preocupan, considerando, por ejemplo, la presencia de caries en más del $30 \%$ de los menores de cinco años y de comunidades en condiciones socioeconómicas desfavorables, pero que son considerados, en su mayoría, poseedores de aseguramiento a través del SGSSS (20).

Los hallazgos hechos gracias a la incorporación de metodologías con enfoques cualitativos, como la de este estudio, evidencian cambios en las percepciones de los padres y los cuidadores de colectivos con similares características. Esto, si se tiene en cuenta 
que en la primera publicación realizada por este equipo de trabajo se señalaban como aspectos relevantes en las representaciones sociales de los cuidadores temas como el acceso a los servicios, el desconocimiento y la falta de motivación a consultar sobre la salud bucal (21).

Actualmente, la población objeto de este estudio, integrada por 183 padres, madres y cuidadores de los Centros Infantiles Institucional 8 horas Fantasías de las Américas acreditan un avance en el conocimiento de temas como el de las patologías y los hábitos asociados con la salud bucal de los menores.

Hoy se puede considerar que hacen parte de la cotidianidad de este colectivo actividades establecidas como prácticas saludables para los menores de cinco años, lo cual se puede ver como un adecuado empoderamiento a partir de programas y políticas que se vienen implementando y aplicando al grupo. En consecuencia, se percibe la existencia de un nivel conceptual y de lenguaje más elevado en temas relacionados con el componente bucal de la salud, integrando hábitos y prácticas saludables como el lavado de manos que contribuyen directamente a mejorar las condiciones de salud en general y, a su vez, en su componente bucal.

El trabajo realizado en la ciudad de Cienfuegos, Cuba, titulado Programa educativo sobre salud bucal para madres con niños en edad preescolar, muestra cómo los procesos basados en educación para la salud derivan en resultados positivos, más aún cuando están ligados a la identificación de necesidades de aprendizaje reconocidas en el diagnóstico inicial (22). Es probable que, si se sostienen las acciones educativas a partir de los hallazgos de las realidades identificadas en las representaciones sociales, como en el caso de los cuidadores de Fantasías de las Américas, las percepciones sean motivo de cambio y se expresen en futuros estudios de seguimiento y hallazgo de necesidades que puedan ser asociados. Esto, a su vez, evidencia la trascendencia que tiene la educación para la salud y la necesidad de renovarse, responder a las necesidades particulares de las poblaciones y ofrecer mayores niveles de profundización. Asimismo, invita a entender a los colectivos antes de presentarles propuestas educativas.

Hoy no es suficiente medir aspectos cuantitativos, menos aún con el potencial de conocimiento, saber y expresión que tienen las comunidades. Debe lograrse integrar y dimensionar las categorías cualitativas, para responder a un llamado evidente, $\mathrm{y}$ que permitan establecer nuevos indicadores que visualicen el proceso salud-enfermedad de las poblaciones.

Los resultados de este trabajo presentan avances importantes en la adherencia a estrategias que se pueden estar llevando a cabo en el marco de acciones colectivas, bien sea por parte de entidades aseguradoras y acciones específicas en dimensiones extramurales, o bien por la intervención de programas orientados al fortalecimiento de la educación en el marco de la promoción de la salud. Según los hallazgos, los padres, las madres y los cuidadores demuestran que son conocedores de los beneficios que hoy tienen con las coberturas en sus entidades aseguradoras. Llama la atención las inquietudes relacionadas con condiciones patológicas establecidas, no solamente con problemas relacionados con caries dental, sino ligados a otros sistemas como la articulación temporomandibular y las lesiones de los tejidos blandos. Sobre esto, aparte del conocimiento identificado, desde unos elementos básicos, se muestran claras necesidades de tener acceso a profesionales con formación especialista, pero que faciliten su acceso en los planes de beneficios que ofrece el sistema. 


\section{Conclusiones}

El aporte de este trabajo, con la identificación de las necesidades expresadas y sentidas por padres y cuidadores, exige que se tengan en cuenta nuevas temáticas para enriquecer los saberes desde las actividades de educación y promoción de la salud. Hoy las comunidades entienden, se inquietan y cuestionan el quehacer clínico, las prácticas y los postulados. Igualmente, propone intervenir, desde una mirada indagadora y retrospectiva, en la que se puedan identificar las fuentes de saberes, las experiencias de salud bucal y el papel que han tenido los gestores de la salud bucal en los espacios comunitarios. Se abre una mirada pluralista en la que todos participan, conocen, discuten y exigen calidad.

\section{Agradecimientos}

Al Centro de Desarrollo Social de la Fundación Universitaria Autónoma de las Américas, como oferente del Programa Buen Comienzo de los Centros Infantiles Institucional 8 horas Fantasías de las Américas; a los padres, a las madres y a los cuidadores; a la Facultad de Odontología y sus estudiantes, por su contribución y compromiso con el desarrollo del estudio.

\section{Referencias bibliográficas}

1. Simarra TJ. Panorama de las políticas públicas de primera infancia en Colombia. Miscelánea Comillas Rev Ciencias Humanas y Soc [Internet]. 2010; 68(132):397-417. Disponible en: http://revistas.upcomillas.es/index.php/miscelaneacomillas/ article/view/844

2. Bernal R. La política de primera infancia en el contexto de la equidad y movilidad social en Colombia. Doc CEDE [Internet]. 2012; 33. Disponible en: http://148.215.2.10/redalyc-seam/articulo. oa? $\mathrm{id}=180020302006$

3. Carbonell OA. La sensibilidad del cuidador y su importancia para promover un cuidado de calidad en la primera infancia. Ciencias Psicológicas. 2013; VII(2):201-207.
4. Balcon A, Pascale M, Borodankova O. La educación y atención a la primera infancia. Comisión Europea; 2014.

5. González Martínez F, Sierra Barrios CC, Morales Salinas LE. Conocimientos, actitudes y prácticas en salud bucal de padres y cuidadores en hogares infantiles, Colombia. Salud Pública Méx. 2011; 53(3).

6. Ministerio de Salud, Colombia. Resolución 00412 del 25 de febrero del 2000. Normas técnicas y guías de atención. Bogotá: Diario Oficial; 2000.

7. Estrategia nacional de atención integral a la primera infancia, De Cero a Siempre. Colombia Disponible en: http://www.deceroasiempre.gov. co/QuienesSomos/Documents/Presentacionestrategia-De-Cero-a-Siempre.pdf

8. Herrera ML, Bernal R. Diagnóstico y recomendaciones para la atención de calidad a la primera infancia en Colombia. Bogotá: Fedesarrollo; 2014.

9. Lineamientos y estándares para la prestación del servicio de atención integral a la primera infancia [Internet]. 2015. Disponible en: http://www.icbf.gov. co/portal/page/portal/Descargas1/AnexoTecnicoContratacion-PrimeraInfanciav2.pdf

10. Arango MC, Jaramillo A, Cruz CA. Prevalencia de caries de la infancia temprana y factores de riesgo asociados en niños de 1 a 5 años en la ciudad de Santiago de Cali. Ustasalud. 2013; 12:118-15.

11. Lineamientos de política pública de salud bucal de Medellín 2013-2022. Medellín: Secretaría de Salud de Medellín, Universidad de Antioquia; 2015. Disponible en: http://www.udea.edu.co/wps/wcm/connect/udea/6f16d8c0-aa89-49f5-8d35-948c1d041c8c/ Lineamientos.pdf?MOD $=$ AJPERES

12. García B, González S, Quiroz A, Velásquez A, Ghiso A. Técnicas interactivas para la investigación social cualitativa [Internet]. Fundación Universitaria Luis Amigó, Medellín, 2002. Disponible en: http://aprendeenlinea.udea.edu.co/lms/moodle/ pluginfile.php/101098/mod_resource/content/0/ tecnicas_interactivas1.pdf

13. Vitorelli Diniz Lima Fagundes K, Almeida Magalhães A, Dos Santos Campos CC, Garcia Lopes Alves C, Mônica Ribeiro P, Mendes MA Hablando de la observación participante en la investigación cualitativa en el proceso saludenfermedad [Internet]. Index de Enfermería. 2014; 75-9. Disponible en: http://dx.doi.org/10.4321/ S1132-12962014000100016

14. Ministerio de Salud. Resolución 8430 de 1993 [Internet]. Disponible en: http://www.unisabana. edu.co/fileadmin/Documentos/Investigacion/comite_de_etica/Res_8430_1993_-_Salud.pdf

15. Escobar-Paucar G, Sosa-Palacio C, Burgos-Gil LM. Representaciones sociales del proceso saludenfermedad bucal en madres gestantes de una población urbana: Medellín, Colombia. Salud Pública de México. 2010; 52:46-51. Disponible en: http:// www.scielosp.org/pdf/spm/v52n1/v52n1a07.pdf

16. Escobar-Paucar G, Sosa-Palacio C, Sánchez-Mejía A. Salud bucal: representaciones sociales en madres gestantes de una población urbana. Medellín, Colombia. Cien Saude Colet [Internet]. 2011; 
16(11):4533-40. Disponible en: http://www.scielo. br/pdf/csc/v16n11/a26v16n11.pdf

17. Hilton IV, Stephen S, Barker JC, Weintraub JA. Cultural factors and children's oral health care: a qualitative study of carers of young children. Community Dent Oral Epidemiol [Internet]. 2007; 35(6):429-38. Disponible en: http://dx.doi. org/10.1111/j.1600-0528.2006.00356.x

18. Hoeft KS, Barker JC, Masterson EE. Urban Mexican-American mothers' beliefs about caries etiology in children. Community Dent Oral Epidemiol [Internet]. 2010;38(3):244-55. Disponible en: http://dx.doi.org/10.1111/j.1600-0528.2009.00528.x

19. Vermaire JH, Hoogstraten J, Van Loveren C, Poorterman JHG, Van Exel NJA. Attitudes towards oral health among parents of 6-year-old children at risk of developing caries. Community Dent Oral Epide- miol [Internet]. 2010; 38(6):507-20. Disponible en: http://dx.doi.org/10.1111/j.1600-0528.2010.00558.x

20. Ministerio de Salud y Protección Social. IV Estudio Nacional de Salud Bucal ENSAB - IV 2013-2014. Bogotá: Ministerio de Salud y Protección Social; 2015. Disponible en: https://www.minsalud.gov. co/sites/rid/Lists/BibliotecaDigital/RIDE/VS/PP/ ENSAB-IV-Situacion-Bucal-Actual.pdf

21. González-Penagos C, Cano-Gómez M, MenesesGómez EJ, Vivares-Builes AM. Percepciones en salud bucal de los niños y niñas. Rev Latinoam Ciencias Soc Niñez y Juv. 2015; 13(2):715-24.

22. Menguez Becerra T, Sexto Delgado N, González Beriau Y. Programa educativo sobre salud bucal para madres con niños en edad preescolar. MediSur. 2009; 7(1):49-53. 